(2) Open Access Full Text Article

ORIGINALRESEARCH

\title{
Association Between Serum Uric Acid Levels and Cognitive Function in Patients with Ischemic Stroke and Transient Ischemic Attack (TIA): A 3-Month Follow-Up Study
}

This article was published in the following Dove Press journal:

Neuropsychiatric Disease and Treatment

\author{
Qi Liu (1D' \\ Xiaoling Liao' \\ Yuesong $\mathrm{Pan}^{2}$ \\ Aoming Jin ${ }^{2}$ \\ Yumei Zhang ${ }^{3}$ \\ 'Department of Neurology, Beijing \\ Tiantan Hospital, Capital Medical \\ University, Beijing, People's Republic of \\ China; ${ }^{2}$ China National Clinical Research \\ Center for Neurological Diseases, \\ Capital Medical University, Beijing, \\ People's Republic of China; ${ }^{3}$ Department \\ of Rehabilitation Medicine, Beijing \\ Tiantan Hospital, Capital Medical \\ University, Beijing, People's Republic of \\ China
}

Purpose: Cognitive impairment is a common complication after stroke and transient ischemic attack (TIA). The relationship between serum uric acid (SUA) and post-stroke cognitive impairment (PSCI) is controversial. This study evaluated the association of different SUA levels in the normal range and PSCI at 3 months.

Patients and Methods: A total of 1523 patients with ischemic stroke/TIA were recruited from the Impairment of Cognition and Sleep (ICONS) subgroup of the China National Stroke Registry-3 (CNSR-3). SUA concentration was assessed at baseline. Global cognitive status was evaluated using the Montreal Cognitive Assessment (MoCA). The main clinical outcome was the incidence of PSCI assessed at 3 months after stroke/TIA. The association between SUA status and the risk of PSCI was assessed with multiple regression models adjusted for potential covariates.

Results: Among the 1523 patients (1391 (91.33\%) stroke patients and 132 (8.67\%) TIA patients), 747 (49.05\%) patients had PSCI at 3 months. Compared to the reference group, there was an increased risk of PSCI in males with SUA levels in the first $(\mathrm{OR}=1.76)$ and fourth quartiles $(\mathrm{OR}=1.47)$. A U-shaped association between SUA levels and the incidence of PSCI with an inflection point of $297 \mathrm{mmol} / \mathrm{L}$ was also found in males. However, there was no association between SUA levels and PSCI in females.

Conclusion: The association between SUA and PSCI differed between males and females. In males, both low and high SUA levels were associated with relatively higher incidences of PSCI, supporting a U-shaped association between SUA levels and PSCI.

Keywords: uric acid, cognitive impairment, stroke, transient ischemic attack, U-shaped curve

\section{Introduction}

Stroke is the second most common cause of morbidity and mortality worldwide. ${ }^{1}$ Cognitive impairment is a common complication after stroke, which is associated with a poor prognosis and imposes a heavy burden on families and society. A metaanalysis published in 2019 found that approximately 38\% of stroke patients developed cognitive dysfunction within 1 year, $26.5 \%$ of whom progressed to dementia. ${ }^{2}$ A Chinese community-based study showed that the overall prevalence of poststroke cognitive impairment (PSCI) was as high as $80.97 \%$, indicating the need to recognize and prevent PSCI in stroke patients. ${ }^{3}$ Several molecular biomarkers have
Correspondence: Yumei Zhang Department of Rehabilitation Medicine, Beijing Tiantan Hospital, Capital Medical University, I I 9\#Nan Si Huan Xi Lu, Beijing, 100070, People's Republic of China Tel +86 |36-9| $40-4 \mid 06$

Email zhangyumei95@aliyun.com
Neuropsychiatric Disease and Treatment 2021:17 99|-999 
been reported to be associated with cognitive decline after stroke, which may improve the diagnostic and prognostic accuracy with regard to PSCI. ${ }^{4}$

Uric acid (UA) is the end product of nucleic acid metabolism, and it can be used as an index to evaluate renal function. ${ }^{5-11}$ Studies have identified the relationship between serum uric acid (SUA) levels and cognition, but the results have been contradictory. Previous studies found that an elevated SUA level was strongly related to vascular dementia, suggesting that SUA might be a risk factor. ${ }^{8-11}$ However, some studies found that UA may have a protective effect on cognition. ${ }^{5-7}$ A similar argument was also made in studies focusing on PSCI. ${ }^{12,13}$

As an important antioxidant and free radical scavenging agent in the human body, UA may influence cognition through its effect on the oxidative stress pathway. However, UA can accelerate the oxidative stress reaction under certain pathological conditions. Furthermore, an antioxidant-prooxidant urate redox shuttle was identified at a level of SUA of $238 \mathrm{mmol} / \mathrm{L} \cdot{ }^{14,15}$ The controversy about the association of SUA with PSCI may be due to the complicated underlying pathophysiological process. Previous studies found that a SUA level $<372 \mathrm{mmol} / \mathrm{L}$ was negatively correlated with the degree of neurological impairment in stroke patients, while the protective effect disappeared when the SUA level was $>372 \mathrm{mmol} / \mathrm{L} .{ }^{16}$ Due to the nonlinear association between the SUA level and stroke, is there an inflection point of SUA with regard to its relationship with the occurrence of PSCI?

Accordingly, our study aimed to explore the association between the SUA level and PSCI, which may help identify potential biomarkers for the detection and prevention of PSCI.

\section{Patients and Methods Study Population}

Patients were identified from the Impairment of Cognition and Sleep (ICONS) study of the China National Stroke Registry-3 (CNSR-3). Details on the ICONS study have been previously published. ${ }^{17}$ Briefly, the ICONS study was a nationwide multicenter prospective registry involving 40 hospitals between August 2015 and March 2018 in China that recruited consecutive acute ischemic stroke (AIS) and TIA patients. Patients in the registry met the following criteria: age older than 18 years; inclusion within 7 days after the onset of in-hospital AIS or TIA; no history of a severe cognitive disorder before stroke; and no concomitant disorders that could interfere with the cognitive evaluation.

Our study aimed to investigate the relationship between SUA levels in the normal range and PSCI at 3 months after onset. We chose patients who had SUA data at baseline and completed the neuropsychological assessments at the 3-month follow-up. We excluded patients with a history of stroke and diagnosed hyperuricemia, which was defined as a SUA level $>420 \mathrm{mmol} / \mathrm{L}$ in males and $>360 \mathrm{mmol} / \mathrm{L}$ in females according to the guidelines. $^{18}$

According to the Helsinki Declaration, this study was approved by the Ethics Committees of Beijing Tiantan Hospital.

\section{Baseline Data Collection}

Patient demographics and history of cognitive risk factors, such as diabetes mellitus, hypertension, smoking and alcohol consumption were collected on admission. Baseline parameters were measured on admission from blood samples, including the levels of SUA, hemoglobin, cholesterol and triglycerides.

\section{Outcome Assessment}

The outcome was the incidence of PSCI at 3 months after AIS or TIA onset. The Montreal Cognitive Assessment (MoCA) was used to assess global cognition. Cognitive impairment was defined as a MoCA score $<25$ when the patient's level of education was $<12$ years; otherwise, it was defined as a MoCA score $<26$ points. ${ }^{19}$

\section{Statistical Analysis}

The data analysis was conducted with SAS 9.4 (SAS Institute Inc, Cary, NC). Continuous variables are represented as the medians and interquartile ranges. Categorical variables are presented as frequencies and percentages. Patients in this study were categorized into 4 groups by SUA quartile and were grouped by sex because there are sex-based differences in SUA levels. Baseline variables were compared among groups with different levels of SUA with the Kruskal-Wallis $H$-test for continuous variables and the chi-square test for categorical variables.

The association of SUA levels with PSCI was analysed by multivariable logistic regression. Adjusted odds ratios (ORs) and their 95\% confidence intervals (CIs) were calculated. Then, 3 models were constructed. Model 1 was adjusted for age, education and body mass index (BMI); model 2 was additionally adjusted for patients' self-reported 
history of diseases; and model 3 was further adjusted for the additional parameters shown in Table 1.

A potential nonlinear relationship of the level of SUA with the risk of PSCI was also evaluated using a logistic regression model with the cubic spline function. The inflection point of the SUA level at which the association began to change was calculated using a recursive algorithm. Then, a piecewise logistic model was applied to both sides of the inflection point. A log likelihood ratio test was used to compare the logistic regression model with the piecewise logistic regression model to determine which model was better suited for describing the association between SUA and PSCI.

\section{Results}

\section{Study Participants and Characteristics (Correlations Between SUA Level and} Baseline Characteristics of PSCI Patients) A total of 2625 patients were enrolled in the ICONS study. We excluded 888 patients with a history of stroke and those missing SUA levels on admission and the MoCA results at the 3-month follow-up. And 153 males and 61 females were excluded for hyperuricemia. Finally, 1523 patients were included in our analysis. (Figure 1) Among these patients, the average age was 61 years (range 50-71), and 1091 (71.635\%) were male.

The socio-demographic and clinical characteristics are shown in Table 1. The ranges of SUA for quartiles 1-4 (Q1-4) were <251, 251-297.3, 297.3-343, > $343 \mathrm{mmol} / \mathrm{L}$ in males and <206, 206-248, 248-282, $>282 \mathrm{mmol} / \mathrm{L}$ in females, respectively. Significant differences in age, BMI, estimated glomerular filtration rate (eGFR), creatinine $(\mathrm{Cr})$, hemoglobin $(\mathrm{Hb})$, hematocrit $(\mathrm{Hct})$, total cholesterol, low-density lipoprotein (LDL), triglycerides (TG), hypertension, diabetes mellitus, and the use of antihypertensive and hypoglycemic agents were observed among the groups with different SUA levels. (Table 1)

\section{Logistic Regression Analysis}

Table 2 shows the 3-month incidence of PSCI across groups with different SUA levels. A total of 747 $(49.05 \%)$ patients had PSCI, as determined by the MoCA, including 496 (45.46\%) males and 251 (58.10\%) females. In males, the incidence of PSCI was the lowest in the Q2 group (38.8\%). Compared with the Q2 group, patients in Q1 had an approximately 1.7-fold higher risk of PSCI at 3 months (55.0\% vs $38.8 \%$; adjusted $\mathrm{OR}=1.76$,
95\% CI 1.22-2.54, P<0.01), whereas those in Q4 had an approximately 1.5 -fold higher risk of PSCI (47.1\% vs $38.8 \%$; adjusted $\mathrm{OR}=1.47,95 \% \mathrm{CI} 1.01-2.15, \mathrm{P}=0.04$ ). There was no significant association between SUA and PSCI in females. (Table 2)

\section{Nonlinear Association Between SUA and the Incidence of $\mathrm{PSCl}$ in Males}

No linear correlation was found between the SUA level and PSCI (male $\mathrm{P}=0.41$, female $\mathrm{P}=0.18$ ). Figure 2 suggests that the association between SUA and PSCI is likely to be nonlinear. A U-shaped association between the SUA level and PSCI was found regardless of sex but was only statistically significant in males $(p<0.01$ in males, $\mathrm{P}=0.81$ in females) (Figure 2), and the inflection point for males was $297 \mathrm{mmol} / \mathrm{L}$ after adjustment for covariates (Table 3). Due to the small sample size, some results did not reach statistical significance.

\section{Discussion}

In this analysis of data from the ICONS study, we found that both low and high SUA levels were associated with an elevated incidence of PSCI in males but not in females. We also found a U-shaped association between the SUA level and the incidence of PSCI regardless of sex. Furthermore, the incidence of PSCI was lowest in males with a SUA level of $297 \mathrm{mmol} / \mathrm{L}$.

Previous studies have shown an association between SUA levels and cognitive dysfunction. ${ }^{5-7}$ However, studies on the associations between SUA levels and PSCI remain scarce, and the results have been contradictory. A study including 197 patients demonstrated that a high level of SUA was related to better cognition after stroke (OR 0.74, 95\% CI 0.54-0.99). ${ }^{[12]}$ In contrast, a recent study pointed out that a higher SUA was an independent risk factor for the development of PSCI after adjusting for conventional risk factors, including age, sex and years of education. ${ }^{13}$

The contradictory results may be attributed to the sample sizes and adjustment for confounders, and these studies explored the possibility of a linear relationship rather than a nonlinear relationship. Our study is the first to find a nonlinear relationship between SUA levels and the incidence of PSCI. The results of our study differed from those of previous studies, and this difference may be attributed to the following reasons: differences in the selection of confounders and outcomes and differences in the subgroup analysis by sex. 


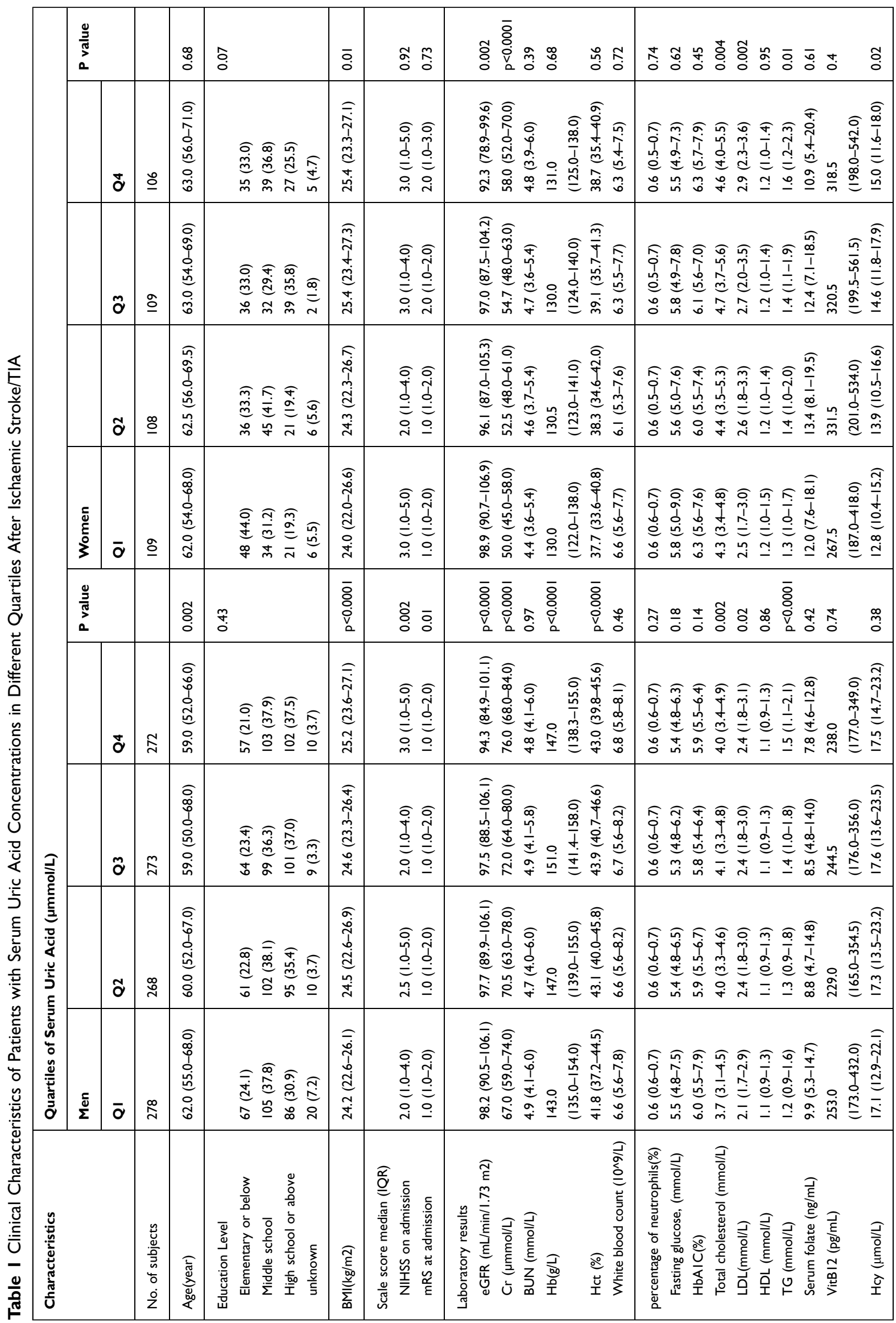




\begin{tabular}{|c|c|c|c|c|}
\hline 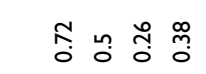 & $\stackrel{\circ}{\infty}$ & กิ่ & กู่ & 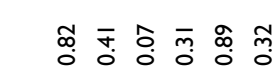 \\
\hline 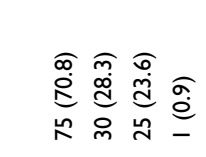 & $\begin{array}{l}\widehat{\alpha} \\
\stackrel{j}{j} \\
\sigma\end{array}$ & $\begin{array}{l}\hat{\dot{e}} \\
\stackrel{0}{0}\end{array}$ & 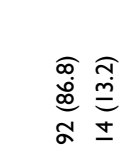 & 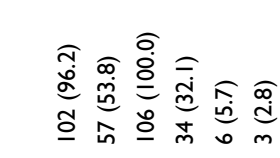 \\
\hline 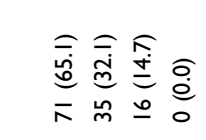 & $\stackrel{\widehat{\infty}}{\stackrel{N}{*}}$ & $\stackrel{\widehat{\sigma}}{\stackrel{\rho}{\dot{\theta}}}$ & 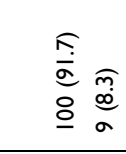 & 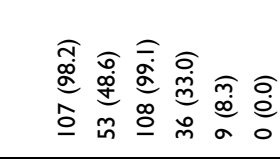 \\
\hline 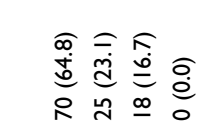 & $\underset{m}{\stackrel{\widehat{\infty}}{d}}$ & $\stackrel{\sigma}{\stackrel{\sigma}{\dot{\theta}}}$ & 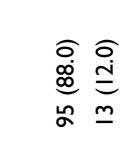 & 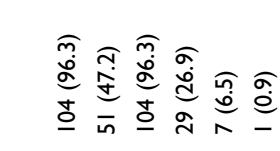 \\
\hline 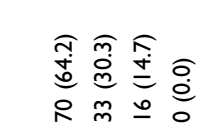 & $\underset{m}{\stackrel{\widehat{D}}{\dot{d}}}$ & $\begin{array}{l}\widehat{O} \\
\stackrel{0}{0} \\
0\end{array}$ & 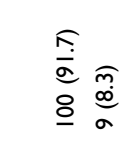 & 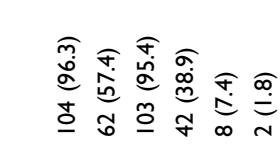 \\
\hline 융 总 & ơ & $\frac{0}{0}$ & $\bar{F}$ & 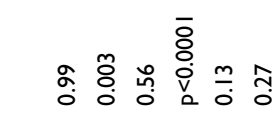 \\
\hline 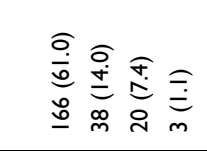 & 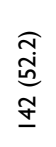 & $\begin{array}{l}\hat{\sigma} \\
\stackrel{d}{d} \\
\stackrel{0}{0}\end{array}$ & 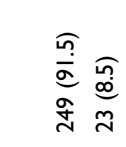 & 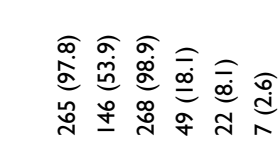 \\
\hline 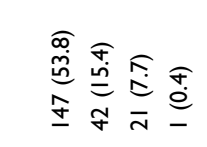 & $\begin{array}{l}\widehat{m} \\
\frac{m}{n} \\
\frac{9}{q}\end{array}$ & 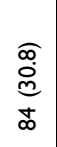 & 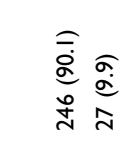 & 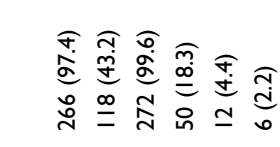 \\
\hline 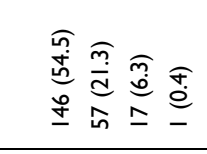 & $\begin{array}{l}\widehat{\widetilde{g}} \\
\underset{j}{\sigma} \\
\underline{m}\end{array}$ & 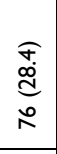 & 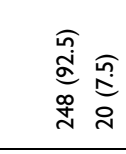 & 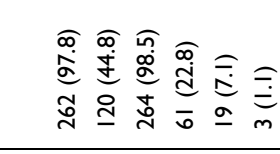 \\
\hline 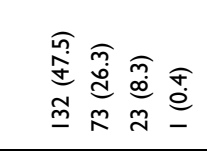 & 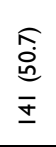 & $\frac{\sigma}{\bar{a}}$ & 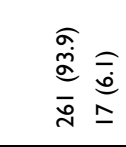 & 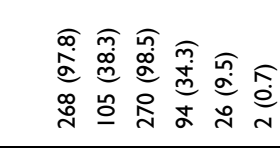 \\
\hline 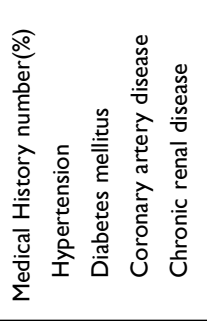 & 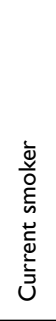 & 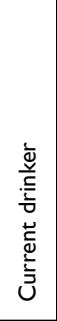 & 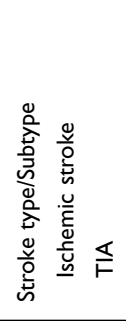 & 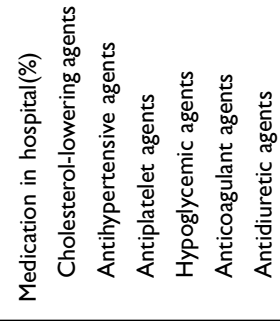 \\
\hline
\end{tabular}


Patients enrolled in the Impairment of Cognition and Sleep quality (ICONS) subgroup of CNSR-III: 2625

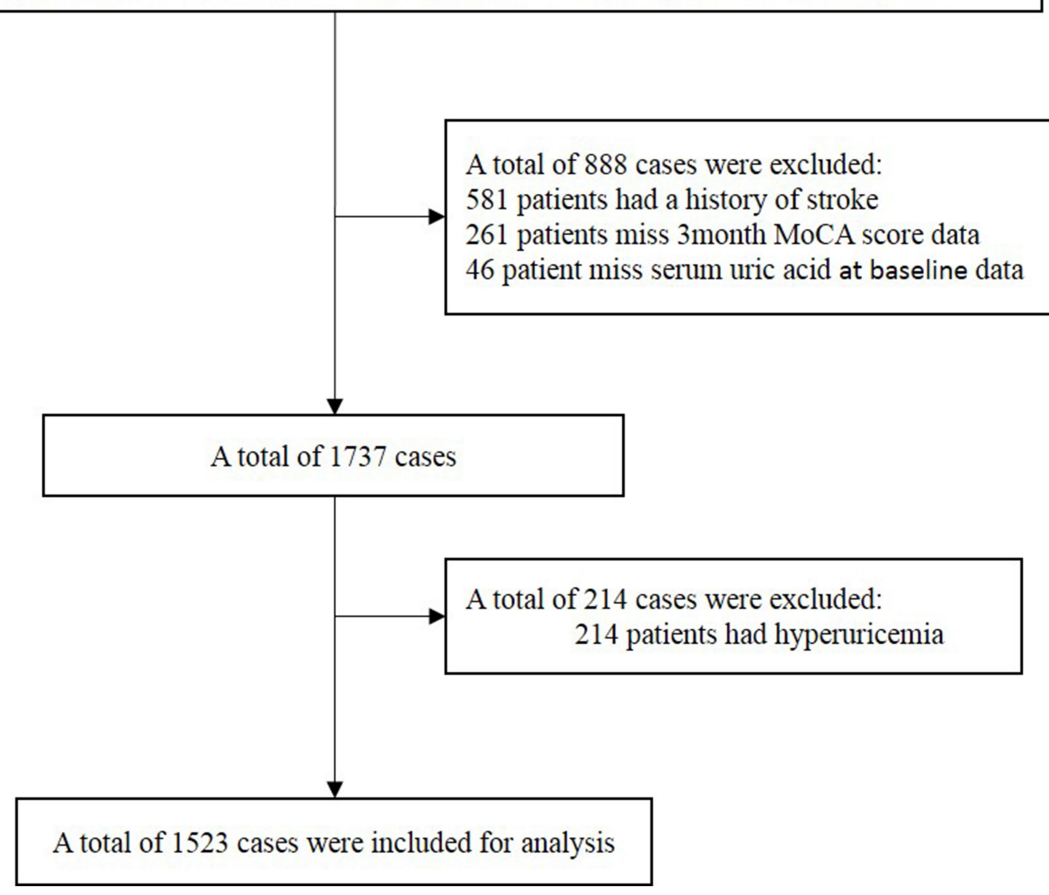

Figure I Flowchart of patients participating in the study.

The mechanism driving this association is still unclear. Previous studies have shown that oxidative stress may contribute to vascular cognitive impairment. ${ }^{20-23}$ As an important antioxidant in the human body, UA may influence cognition through its effect on the oxidative stress pathway. SUA can scavenge free radicals, such as hydroxyl radicals, hydrogen peroxide and peroxynitrite, and inhibit some oxidation reactions, such as lipid peroxidation. ${ }^{24}$ A previous study provided evidence that exogenously administered UA exerts neuroprotective effects in animal models of transient focal brain ischemia by suppressing the accumulation of reactive oxygen species (ROS). ${ }^{25}$

Table 2 Risk of PSCl at 3 Months After Stroke/TIA Stratified by Serum Uric Acid Levels

\begin{tabular}{|c|c|c|c|c|c|c|c|c|c|}
\hline Outcomes & \multirow[t]{2}{*}{ SUA } & \multirow[t]{2}{*}{ No. } & \multirow[t]{2}{*}{ Events, $\mathrm{n}(\%)$} & \multirow{2}{*}{$\begin{array}{l}\text { Model I } \\
\begin{array}{l}\text { Adjusted HR } \\
(95 \% \mathrm{Cl})\end{array}\end{array}$} & \multirow[t]{2}{*}{ p value } & \multirow{2}{*}{$\begin{array}{l}\text { Model } 2 \\
\text { Adjusted HR } \\
(95 \% \mathrm{Cl})\end{array}$} & \multirow[t]{2}{*}{ p value } & \multirow{2}{*}{$\begin{array}{l}\text { Model } 3 \\
\text { Adjusted HR } \\
(95 \% \mathrm{Cl})\end{array}$} & \multirow[t]{2}{*}{ p value } \\
\hline Male & & & & & & & & & \\
\hline & QI & 278 & $153(55.0)$ & $1.80(1.27,2.56)$ & $<0.01$ & $1.82(1.28,2.606)$ & $<0.01$ & $1.76(1.22,2.54)$ & 0.003 \\
\hline & Q2 & 268 & $104(38.8)$ & Ref & & & & Ref & \\
\hline & Q3 & 273 & III (40.7) & I.II $(0.78,1.58)$ & 0.58 & I.II $(0.77$, I.58) & 0.58 & I.II $(0.77$, I.6I) & 0.58 \\
\hline & Q4 & 272 & I $28(47.1)$ & I.45 (I.02, 2.07) & 0.04 & I.45 (I.0I, 2.07) & 0.04 & $1.47(1.01,2.15)$ & 0.05 \\
\hline \multirow[t]{5}{*}{ Female } & & & & $\begin{array}{l}\text { Adjusted HR } \\
(95 \% \mathrm{CI})\end{array}$ & p value & $\begin{array}{l}\text { Adjusted HR } \\
(95 \% \mathrm{CI})\end{array}$ & & $\begin{array}{l}\text { Adjusted HR } \\
(95 \% \mathrm{CI})\end{array}$ & \\
\hline & QI & 109 & $65(59.6)$ & I.I $3(0.64,1.99)$ & 0.68 & $1.20(0.67,2.14)$ & 0.54 & I.38 $(0.76,2.53)$ & 0.29 \\
\hline & Q2 & 108 & $59(54.6)$ & Ref & & Ref & & Ref & \\
\hline & Q3 & 109 & $65(59.6)$ & I.24 $(0.70,2.20)$ & 0.45 & I.28 (0.7I, 2.30) & 0.41 & I.44 $(0.78,2.68)$ & 0.25 \\
\hline & Q4 & 106 & $62(58.5)$ & $1.13(0.64,1.99)$ & 0.67 & $1.17(0.65,2.10)$ & 0.61 & $1.19(0.64,2.21)$ & 0.59 \\
\hline
\end{tabular}

Notes: In Males: Model I: age, BMI, education; Model 2: model I+ hypertension, diabetes mellitus, dyslipidemia, coronary artery disease, current smoking, and current alcohol consumption; Model 3: model 2+ urinary protein, eGFR, white blood cell count, percentage of neutrophils, TG, LDL, total cholesterol, Hb and Hct.

In Females: Model I: age, BMI, education; Model 2: model I+ hypertension, diabetes mellitus, dyslipidemia, coronary artery disease, current smoking and current alcohol consumption; Model 3: model 2+ urinary protein, eGFR, white blood cell count, percentage of neutrophils, TG, LDL and total cholesterol. 

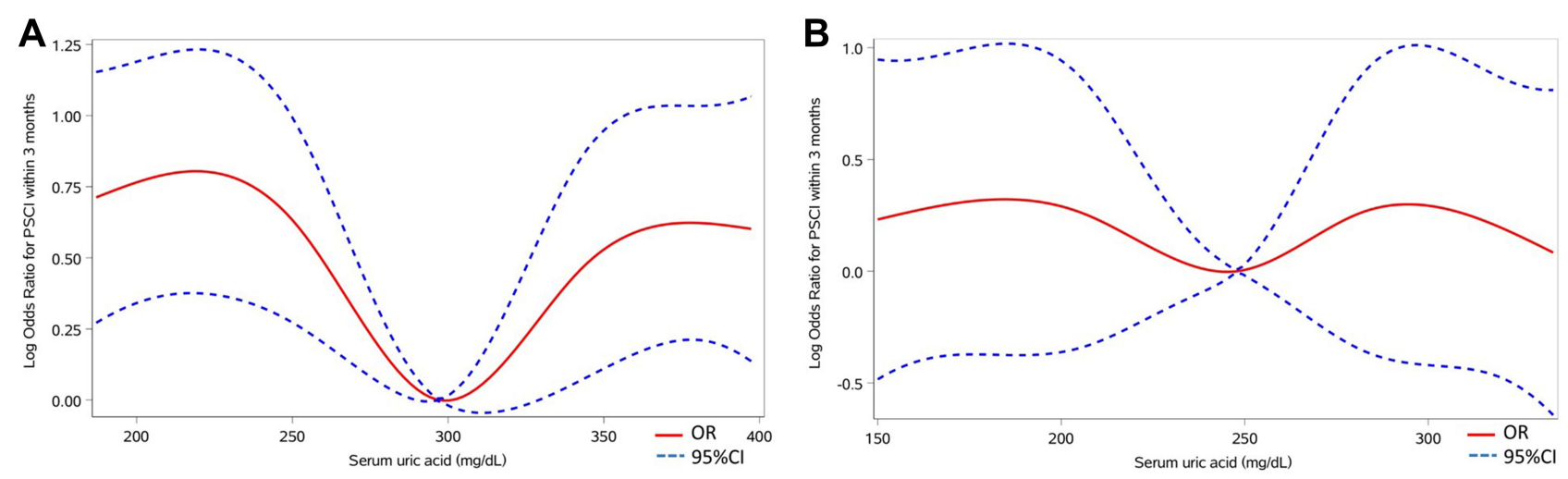

Figure 2 Adjusted odds ratios for PSCl according to SUA levels. Adjusted odds ratios for $\mathrm{PSCl}$ according to SUA levels in males (A) and females (B). The red line indicates the adjusted hazard ratio, and the blue lines indicate the $95 \%$ confidence intervals.

While UA has beneficial antioxidant properties, its production in tissues can release toxic pro-oxidants under pathologic conditions such as ischemia. A previous study found that the antioxidant function of UA was converted into an oxidative function at a serum concentration of $238 \mathrm{mmol} / \mathrm{L} .{ }^{14}$ Furthermore, SUA can chelate transition metals and stimulate the production of inflammatory mediators (such as monocyte chemokine 1, hypersensitive C-reactive protein, interleukin-1, interleukin-6, and tumor necrosis factor), thus further aggravating the oxidative stress response. $^{26,27}$

Moreover, elevated SUA levels also have well-known associations with white matter hyperintensities $(\mathrm{WMH}),{ }^{28,29}$ atherosclerosis, ${ }^{30}$ and arterial stiffness, ${ }^{31}$ which increase the risk of cognitive impairment. ${ }^{32,33}$ The complex biochemical functions of SUA may lead to a nonlinear relationship between SUA and the incidence of PSCI.

However, our study did not find a relationship between SUA and the incidence of PSCI in females, which may be due to the small sample size of the female group. Previous studies also identified sex-based differences. ${ }^{34-36}$ One study suggested that sex-based differences in neural networks may explain this finding and found that men with hyperuricemia were more likely to have spontaneous brain activity changes than women on resting-state functional magnetic resonance imaging. ${ }^{37}$ In addition, we hypothesize that estrogen in females may exert protective effects on blood vessels and thus can attenuate the effect of UA on oxidative stress reactions.

Our study included 1523 patients, and the data from this relatively larger sample size support a relationship between SUA and PSCI. The incidence of PSCI is the highest 3 months after stroke. ${ }^{32}$ By ensuring a sufficient follow-up period, we improved the detection rate of PSCI, which is a long-term complication of stroke. In addition, the relationship of SUA with PSCI in both sexes was explored.

Our study had several limitations. First, the SUA levels were only measured at baseline. A recent study found that SUA elevated 3.41 times in the acute phase of ischemic stroke compared to healthy individuals, and fluctuations in SUA levels might influence the outcome. ${ }^{38}$ Second, fewer women were included in the study than men, which might have affected the results of the correlation analysis. Third, indicators related to SUA level and cognition, such as the

Table 3 Analysis of the Threshold Effect of Serum Uric Acid on PSCl at 3 Months in Males

\begin{tabular}{|l|l|l|l|}
\hline & Model I & Model 2 & Model 3 \\
\hline Inflection point & 297 & 297 & 297 \\
\hline$<297$ & $0.993(0.988,0.997), 0.0023$ & $0.993(0.988,0.997), 0.0017$ & $0.993(0.988,0.998), 0.0077$ \\
\hline$>297$ & $1.004(0.998,1.009), 0.1786$ & $1.003(0.998,1.009), 0.2240$ & $1.003(0.997,1.009), 0.2843$ \\
\hline p for log likelihood ratio test & $<0.001$ & $<0.001$ & $<0.001$ \\
\hline
\end{tabular}

Notes: Model I: age, BMI, education; Model 2: model I+ hypertension, diabetes mellitus, dyslipidemia, coronary artery disease, current smoking, and current alcohol consumption; Model 3: model 2+ urinary protein, eGFR, white blood count, percentage of neutrophils (GRA\%), TG, LDL, total cholesterol, Hb and Hct. 
use of diuretics and level of C-reactive protein, were not included.

\section{Conclusion}

Our study showed that both low and high SUA levels were associated with an elevated risk of PSCI. A SUA level of $297 \mathrm{mmol} / \mathrm{L}$ in males might be considered safe with respect to the risk of PSCI. Our study provides a basis for exploring biomarkers for the early prediction and prevention of PSCI. Further study is necessary to elucidate the actual role of SUA and the potential underlying biological mechanisms.

\section{Abbreviations}

TIA, transient ischemic attack; SUA, serum uric acid; PSCI, post-stroke cognitive impairment; ICONS, Impairment of Cognition and Sleep; CNSR-3, China National Stroke Registry-3; MoCA, Montreal Cognitive Assessment; UA, Uric acid; AIS, acute ischemic stroke; ORs, odds ratios; CIs, confidence intervals; BMI, body mass index; eGFR, estimated glomerular filtration rate; $\mathrm{Cr}$, creatinine; $\mathrm{Hb}$, hemoglobin; Hct, hematocrit; LDL, low-density lipoprotein; TG, triglycerides; ROS, reactive oxygen species; WMH, white matter hyperintensities.

\section{Data Sharing Statement}

Data are available on reasonable request.

\section{Ethics Approval}

This study was approved by the medical Ethics Committee of Beijing Tiantan Hospital (No. KY2015-001-01). All the study participants provided informed consent to take part in this study, in accordance with the Declaration of Helsinki.

\section{Consent for Publication}

Not required.

\section{Acknowledgments}

We appreciate the centers and all the members participated in the ICONS study. We would also like to thank Yongjun Wang and Jiong Shi for academic guidance.

\section{Author Contributions}

All authors contributed to data analysis, drafting or revising the article, have agreed on the journal to which the article will be submitted, gave final approval of the version to be published, and agree to be accountable for all aspects of the work.

\section{Funding}

This work was granted by National Key R\&D Program of China (2018YFC1312903), National Science and Technology Major Project (2017ZX09304018), Beijing Municipal Science \& Technology Commission (D171100003017002, Z181100001818001) and National Natural Science Foundation of China (81972144).

\section{Disclosure}

The authors report no conflicts of interest in this work.

\section{References}

1. Gorelick PJ. The global burden of stroke: persistent and disabling. Lancet Neurol. 2019;18(5):417-418. doi:10.1016/s1474-4422(19) 30030-4

2. Merriman N, Sexton E, McCabe G, et al. Addressing cognitive impairment following stroke: systematic review and meta-analysis of non-randomised controlled studies of psychological interventions. BMJ Open. 2019;9(2):e024429. doi:10.1136/bmjopen-2018-024429

3. Qu Y, Zhuo L, Li N, et al. Prevalence of post-stroke cognitive impairment in china: a community-based, cross-sectional study. PLoS One. 2015;10(4):e0122864. doi:10.1371/journal.pone.0122864

4. Zhang X, Bi X. Post-stroke cognitive impairment: a review focusing on molecular biomarkers. J Mol Neurosci. 2020;70(8):1244-1254. doi:10.1007/s12031-020-01533-8

5. Xu Y, Wang Q, Cui R, et al. Uric acid is associated with vascular dementia in Chinese population. Brain Behav. 2017;7:2. doi:10.1002/ brb3.617

6. Zhang J, Tang L, Hu J, Wang Y, Xu YJB. behavior: uric acid is associated with cognitive impairment in the elderly patients receiving maintenance hemodialysis-A two-center study. 2020;10(3):e01542.

7. Scheepers L, Jacobsson LTH, Kern S, et al. Urate and risk of Alzheimer's disease and vascular dementia: a population-based study. Alzheimers Dement. 2019;15(6):754-763. doi:10.1016/j.jalz.20 19.01.014

8. Wang T, Sun Z-W, Shao L-Q, et al. Diagnostic values of serum levels of homocysteine and uric acid for predicting vascular mild cognitive impairment in patients with cerebral small vessel disease. Med Sci Monitor. 2017;23:2217-2225. doi:10.12659/msm.901652

9. Schretlen D, Inscore A, Jinnah H, et al. Serum uric acid and cognitive function in community-dwelling older adults. Neuropsychology. 2007;21(1):136-140. doi:10.1037/0894-4105.21.1.136

10. Verhaaren B, Vernooij M, Dehghan A, et al. The relation of uric acid to brain atrophy and cognition: the Rotterdam Scan Study. Neuroepidemiology. 2013;41(1):29-34. doi:10.1159/000346606

11. Serdarevic N, Stanciu AE, Begic L, et al. Serum uric acid concentration in patients with cerebrovascular disease (Ischemic stroke and vascular dementia). Med Archives. 2020;74(2):95-99. doi:10.5455/ medarh.2020.74.95-99

12. Ran F, Liu F, Zhang Y, et al. Serum uric acid and high-sensitivity C-reactive protein as predictors of cognitive impairment in patients with cerebral infarction. Dement Geriatr Cogn Disord. 2020:1-8. doi:10.1159/000507806.

13. Sun J, Lv X, Gao X, et al. The association between serum uric acid level and the risk of cognitive impairment after ischemic stroke. Neurosci Lett. 2020;734:135098. doi:10.1016/j.neulet.2020.135098

14. Zhu A, Zou T, Xiong G, et al. Association of uric acid with traditional inflammatory factors in stroke. Int $J$ Neurosci. 2016;126 (4):335-341. doi:10.3109/00207454.2015.1015723 
15. Hayden M, Tyagi SJN. metabolism. Uric acid: a new look at an old risk marker for cardiovascular disease, metabolic syndrome, and type 2 diabetes mellitus: the urate redox shuttle. Nutri Metabol. 2004;1 (1):10. doi:10.1186/1743-7075-1-10

16. Wang R, Zhong Y, Zhou Q, et al. Relationship between uric acid level and severity of acute primary cerebral infarction: a cross-sectional study. 2020;2020:2310307. doi:10.1155/2020/2310307

17. Wang Y, Liao X, Wang C, et al. Impairment of cognition and sleep after acute ischemic stroke or transient ischemic attack in Chinese patients: design, rationale and baseline patient characteristics of a nationwide multicenter prospective registry. Stroke Vasc Neurol. 2020. doi:10.1136/svn-2020-000359

18. Dalbeth N, Bardin T, Doherty M, et al. Discordant American College of Physicians and international rheumatology guidelines for gout management: consensus statement of the Gout, Hyperuricemia and Crystal-Associated Disease Network (G-CAN). Nat Rev Rheumatol. 2017;13(9):561-568. doi:10.1038/nrrheum.2017.126

19. Nasreddine Z, Phillips N, Bédirian V, et al. The Montreal Cognitive Assessment, MoCA: a brief screening tool for mild cognitive impairment. J Am Geriatrics Soc. 2005;53(4):695-699. doi:10.1111/ j.1532-5415.2005.53221.x

20. Seet R, Kasiman K, Gruber J, et al. Is uric acid protective or deleterious in acute ischemic stroke? A prospective cohort study. Atherosclerosis. 2010;209(1):215-219. doi:10.1016/j.atherosclerosis. 2009.08.012

21. Dong Y, Kataoka K, Toyama K, et al. Attenuation of brain damage and cognitive impairment by direct renin inhibition in mice with chronic cerebral hypoperfusion. Hypertension (Dallas, Tex.: 1979). 2011;58(4):635-642. doi:10.1161/hypertensionaha.111.173534

22. Iadecola CJN. The pathobiology of vascular dementia. 2013;80 (4):844-866. doi:10.1016/j.neuron.2013.10.008

23. Bennett S, Grant M, Aldred SJ. Oxidative stress in vascular dementia and Alzheimer's disease: a common pathology. J Alzheimer's Dis. 2009;17(2):245-257. doi:10.3233/jad-2009-1041

24. Amaro S, Llull L, Renú A, et al. Uric acid improves glucose-driven oxidative stress in human ischemic stroke. Ann Neurol. 2015;77 (5):775-783. doi:10.1002/ana.24378

25. Onetti Y, Dantas A, Pérez B, et al. Middle cerebral artery remodeling following transient brain ischemia is linked to early postischemic hyperemia: a target of uric acid treatment. Am J Physiol Heart Circulatory Physiol. 2015;308(8):H862-74. doi:10.1152/ajpheart.00 001.2015

26. Polidori M, Mattioli P, Aldred S, et al. Plasma antioxidant status, immunoglobulin $\mathrm{g}$ oxidation and lipid peroxidation in demented patients: relevance to Alzheimer disease and vascular dementia. Dementia Geriatric Cogn Disord. 2004;18(3-4):265-270. doi:10.11 59/000080027
27. Dawson J, Walters MJB. Uric acid and xanthine oxidase: future therapeutic targets in the prevention of cardiovascular disease? $\mathrm{Br}$ $J$ Clin Pharmacol. 2006;62(6):633-644. doi:10.1111/j.1365-2125.20 06.02785.x

28. Sarfo F, Akassi J, Antwi N, et al. Highly prevalent hyperuricemia is associated with adverse clinical outcomes among ghanaian stroke patients: an observational prospective study. Ghana Med J. 2015;49 (3):165-172. doi:10.4314/gmj.v49i3.7

29. Kamei K, Konta T, Hirayama A, et al. Associations between serum uric acid levels and the incidence of nonfatal stroke: a nationwide community-based cohort study. Clin Exp Nephrol. 2017;21 (3):497-503. doi:10.1007/s10157-016-1311-7

30. Kang D, Park S, Lee I, et al. Uric acid-induced C-reactive protein expression: implication on cell proliferation and nitric oxide production of human vascular cells. J Ame Soci Nephrol. 2005;16 (12):3553-3562. doi:10.1681/asn.2005050572

31. Rebora P, Andreano A, Triglione N, et al. Association between uric acid and pulse wave velocity in hypertensive patients and in the general population: a systematic review and meta-analysis. Blood Pressure. 2020;29(4):220-231. doi:10.1080/08037051.2020.1735929

32. Jh S, et al. Post-Stroke Cognitive Impairment. 2014;2(8):80. doi:10.3978/j.issn.2305-5839.2014.08.05

33. Li X, Lyu P, Ren Y, An J, Dong YJ. Arterial stiffness and cognitive impairment. $J$ Neurol Sci. 2017;380:1-10. doi:10.1016/j.jns.20 17.06 .018

34. Baena CP, Suemoto CK, Barreto SM, et al. Serum uric acid is associated with better executive function in men but not in women: baseline assessment of the ELSA-Brasil study. Exp Gerontol. 2017;92:82-86. doi:10.1016/j.exger.2017.03.010

35. Niu W, Yang $\mathrm{H}$, Lu C. The relationship between serum uric acid and cognitive function in patients with chronic heart failure. BMC Cardiovasc Disord. 2020;20(1):381. doi:10.1186/s12872-020-01 666-z

36. Vannorsdall TD, Kueider AM, Carlson MC, et al. Higher baseline serum uric acid is associated with poorer cognition but not rates of cognitive decline in women. Exp Gerontol. 2014;60:136-139. doi:10.1016/j.exger.2014.10.013

37. Lin L, Zheng LJ, Joseph Schoepf U, et al. Uric acid has different effects on spontaneous brain activities of males and females: a cross-sectional resting-state functional MR Imaging Study. Front Neurosci. 2019;13:763. doi:10.3389/fnins.2019.00763

38. Fatima T, Iftikhar S, IJJotCoP Q, S-p: JCPSP. Association between Hyperuricemia and Ischemic Stroke: a Case-Control Study. J Coll Phys Surg Pak. 2020;30(8):853-856. doi:10.29271/jcpsp.2020. 08.853
Neuropsychiatric Disease and Treatment

\section{Publish your work in this journal}

Neuropsychiatric Disease and Treatment is an international, peerreviewed journal of clinical therapeutics and pharmacology focusing on concise rapid reporting of clinical or pre-clinical studies on a range of neuropsychiatric and neurological disorders. This journal is indexed on PubMed Central, the 'PsycINFO' database and CAS, an is the official journal of The International Neuropsychiatric Association (INA). The manuscript management system is completely online and includes a very quick and fair peer-review system, which is all easy to use. Visit http://www.dovepress.com/testimonials.php to read real quotes from published authors. 